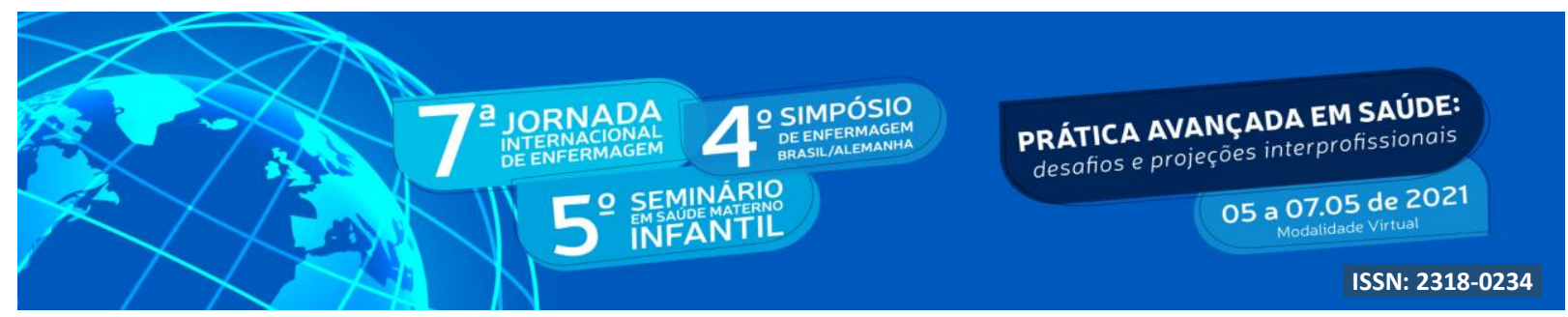

DOI: http://doi.org/10.48195/jie2021-150

\title{
LIGA ACADÊMICA DE ENFERMAGEM OBSTÉTRICA COMO FERRAMENTA NA FORMAÇÃO DO ENFERMEIRO: UM RELATO DE EXPERIÊNCIA ${ }^{1}$
}

\section{Bruna Matias Alcântara²; Maria Débora Silva de Carvalho³ ${ }^{3}$ Evelyn Silva de Sousa ${ }^{4}$; Ana Luiza Santos Quirino5; Leandro da Silva de Medeiros'; Dirce Stein Backes?.}

\begin{abstract}
RESUMO
Objetivo: descrever a experiência de estudantes de Enfermagem referente a participação em uma Liga Acadêmica de Enfermagem Obstétrica. Método: trata-se de um estudo descritivo, do tipo relato de experiência, desenvolvido por discentes de enfermagem no período de janeiro a março de 2021. Resultados: durante as quatro aulas ofertadas aos ligantes, foi possível observar e refletir sobre a importância do enfermeiro no exercício da obstetrícia. Conclusão: conclui-se que as Ligas acadêmicas se estabelecem como um instrumento de grande impacto na formação e no aprofundamento dos conhecimentos de um futuro profissional da enfermagem.
\end{abstract}

Palavras-chave: Educação em Saúde; Enfermagem; Liga Acadêmica; Obstetrícia.

\begin{abstract}
Objective: describe the experience of Nursing students regarding their participation in an Academic Obstetric Nursing League. Method: this is a descriptive study, of the experience report type, developed by nursing students from January to March 2021. Results: During the four classes offered to the league members, it was possible to observe and reflect on the importance of the nurse in obstetrics. Conclusion: It is concluded that the academic leagues are established as an instrument of great impact in the formation and the deepening of the knowledge of future nursing professionals.
\end{abstract}

Key Words: Health education; Nursing; Academic League; Obstetrics.

\footnotetext{
${ }^{1}$ Relato de experiência.

2 Estudante do Curso de Enfermagem. Universidade Federal do Rio Grande do Norte. Membro da Liga Acadêmica de Enfermagem Obstétrica (LAEO/UFRJ). E-mail: brumatias21@ gmail.com

3 Estudante do Curso de Enfermagem. Universidade Federal do Rio Grande do Norte. Membro da Liga Acadêmica de Enfermagem Obstétrica (LAEO/UFRJ). E-mail: debscarvalho88@gmail.com

${ }^{4}$ Estudante do Curso de Enfermagem. Universidade Federal do Rio Grande do Norte. Membro da Liga Acadêmica de Enfermagem Obstétrica (LAEO/UFRJ).E-mail: evelyntdle@ gmail.com

${ }^{5}$ Estudante do Curso de Enfermagem. Universidade Federal do Rio Grande do Norte. Membro da Liga Acadêmica de Enfermagem Obstétrica (LAEO/UFRJ). E-mail: luizasantos699@gmail.com

${ }^{6}$ Estudante do Curso de Enfermagem. Universidade Franciscana. Membro da Liga Acadêmica de Enfermagem Obstétrica (LAEO/UFRJ).E-mail: leandro.medeiros@ufn.edu.br

${ }^{7}$ Orientadora. Doutora em Enfermagem. Docente do Curso de Enfermagem e Coordenadora do Mestrado Profissional Saúde Materno Infantil da Universidade Franciscana. E-mail: backesdirce@ufn.edu.br
} 


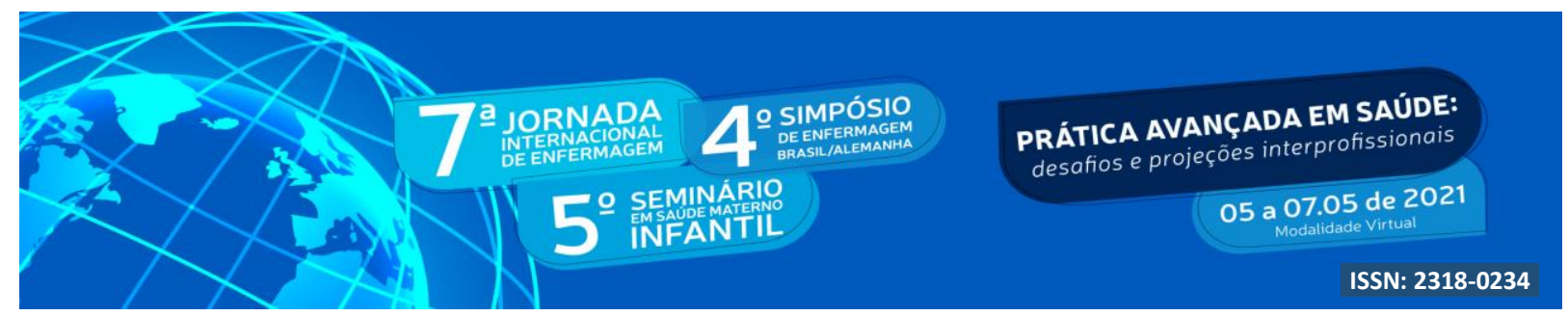

\section{INTRODUÇÃ̃O}

A Lei de Diretrizes e Bases da Educação Nacional (LDB), criada em 1996, dispõe que as universidades devem abranger medidas e ações de ensino, pesquisa e extensão, de modo a proporcionar vivências que levem os alunos a reflexão e aprimoramento de suas condutas, de forma individual e coletiva (BRASIL, 1996; CAVALCANTE et al., 2018). Neste cenário, inserem-se as ligas acadêmicas (LA), que podem ser definidas como associações protagonizadas por estudantes, sob a coordenação de um professor orientador, com o objetivo de aprofundar o conhecimento acerca de determinada temática, preenchendo as lacunas existentes na sociedade (SILVA; FLORES, 2015).

Historicamente, as ligas acadêmicas formaram-se nas faculdades de medicina, no início do século 20, devido às demandas para combater as altas taxas de morbimortalidade existentes, especialmente, por hanseníase e tuberculose (SILVA; FLORES, 2015). Posteriormente, no entanto, ocorreu uma expansão para os demais cursos da área da saúde, tanto pelas necessidades de aprendizagem existentes também em outras graduações, quanto pelo potencial transformador que as LA possuem, contribuindo na formação de profissionais humanísticos, críticos e reflexivos, capazes de atuar em todos os níveis de atenção à saúde (CAVALCANTE et al., 2018).

Dentre as áreas abrangidas pelas ligas acadêmicas, destaca-se a obstetrícia, especialidade que versa sobre a saúde reprodutiva da mulher, investigando as mudanças fisiológicas e patológicas presentes na gestação, parto e puerpério, bem como as alterações culturais, sociais e emocionais envolvidas na chegada de um novo ser (CAETANO et al., 2016). O enfermeiro, neste contexto, é um profissional capacitado para realizar todo o prénatal de risco habitual, como disposto pelo decreto $\mathrm{n}^{\circ}$ 94.406/87 (OLIVEIRA; BARBOSA; MELO, 2016).

Para isso, realiza estratégias de promoção à saúde, prevenção de agravos e o tratamento de condições que possam influenciar o período de gestação, parto e puerpério (LEITE et al., 2020). Nesse sentido, uma liga acadêmica de enfermagem obstétrica visa o aperfeiçoamento da prática de enfermagem na assistência à gestante e a parturiente, que, por meio do tripé ensino-pesquisa-extensão, forma enfermeiros com competências, habilidades e práticas acolhedoras ao binômio mãe-filho (SILVA; AMPARO; SANTOS, 2017). 


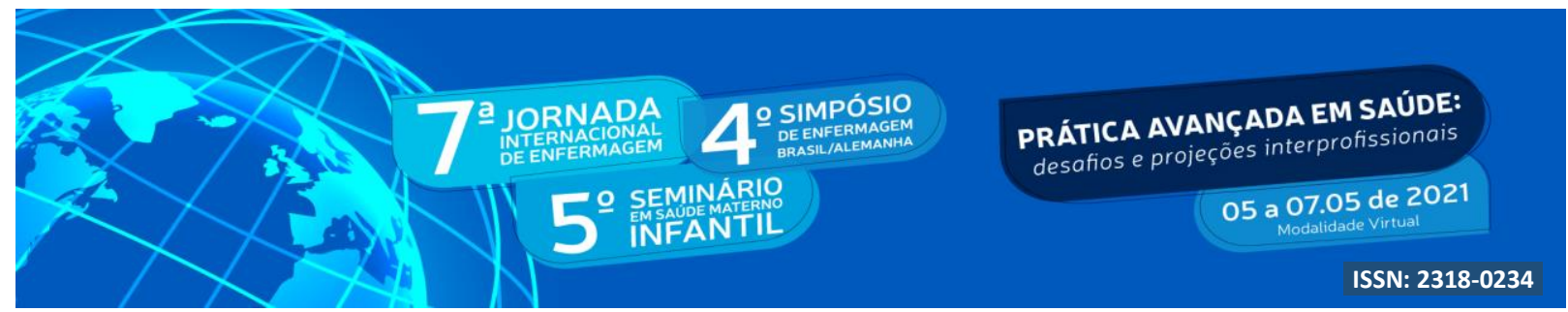

Dessa forma, o presente estudo justifica-se por relatar como uma liga acadêmica de enfermagem obstétrica pode contribuir para a formação de enfermeiros capacitados a prestar uma assistência embasada em evidências, humanizada e resolutiva à mulher durante todo o ciclo gravídico-puerperal, com práticas que promovam melhores desfechos perinatais e a saúde materno-fetal.

\section{OBJETIVO}

Descrever a experiência de estudantes de Enfermagem referente a participação em uma Liga Acadêmica de Enfermagem Obstétrica.

\section{METODOLOGIA}

Trata-se de um estudo descritivo, do tipo relato de experiência que, de acordo com Ferreira e Faller (2020), é um texto que descreve, precisamente, uma dada experiência a fim de contribuir, de forma relevante, para sua área de atuação. Segundo estes, é a descrição que um autor ou uma equipe fazem de uma vivência profissional tida como exitosa ou não, mas que permita a discussão, a troca e a proposição de ideias.

O estudo foi desenvolvido por estudantes de duas instituições de ensino superior (IES), sendo quatro estudantes de enfermagem da Universidade Federal do Rio Grande do Norte (UFRN) e um estudante de enfermagem da Universidade Franciscana (UFN). O relato baseia-se na experiência dos estudantes quanto à participação na função de ligantes da Liga Acadêmica de Enfermagem Obstétrica da Universidade Federal do Rio de Janeiro (LAEO/UFRJ).

A Liga Acadêmica de Enfermagem Obstétrica da UFRJ iniciou as atividades em agosto de 2019, com ações de integração entre ensino, pesquisa e extensão na área de enfermagem obstétrica da Escola de Enfermagem Anna Nery. Atualmente, devido à pandemia da COVID-19, os encontros precisaram ocorrer de forma remota, fazendo com que a instituição possibilitasse que estudantes de todo Brasil integrassem na LAEO, a partir de um processo seletivo (EDITAL 001/2021) divulgado pela IES.

Dessa forma, a experiência em tela, aconteceu entre o período de janeiro a março de 2021 a partir das ações realizadas pela liga, tais como aulas inaugurais, palestras e outras. 


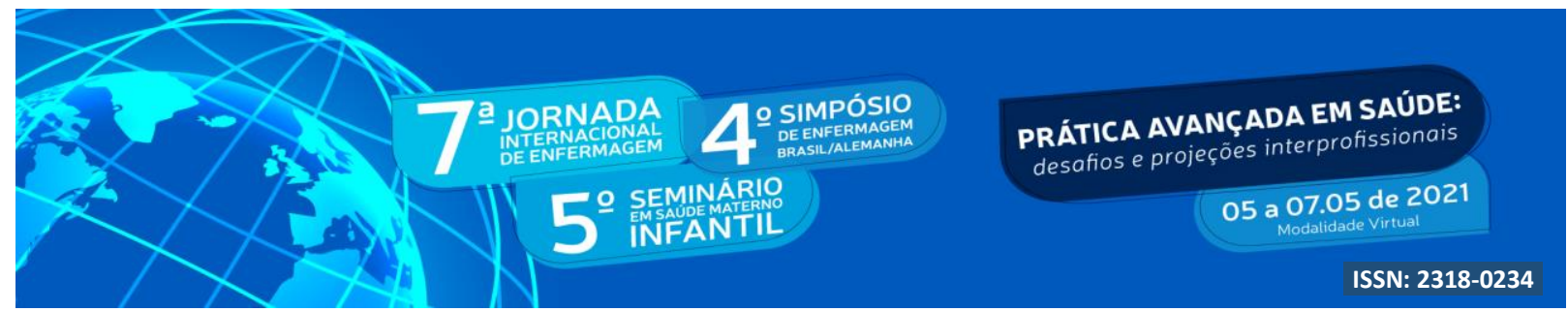

Ressalta-se que todos os encontros ocorreram de modo virtual, pelas plataformas Youtube e Jisi Meet., com base em cronograma de atividades semestrais.

Sob um primeiro enfoque, o processo seletivo para a LAEO foi amplamente divulgado pelas mídias digitais e, dessa forma, permitiu um grande alcance não só para os alunos da própria instituição, mas para discentes de todo o país. Em relação ao número de vagas, foram ofertadas cem ao todo, sendo setenta para alunos da própria Universidade Federal do Rio de Janeiro e trinta para alunos de enfermagem das demais instituições do Brasil.

Paralelo a isso, teve-se como pré-requisitos: ser estudante de enfermagem; ter obtido aprovação na disciplina de fisiologia; e participar da aula inaugural. É importante salientar ainda que, a seleção concretizou-se por ordem de inscrição e os certificados ao final do projeto só seriam entregues aos ligantes com assiduidade de, pelo menos, $75 \%$. Tal assiduidade foi medida por testes lançados antes e após as aulas síncronas, que duravam em torno de duas horas, com a finalidade de avaliar o grau de conhecimento do ligante e verificar sua presença.

\section{RESULTADOS E DISCUSSÃO}

A formação do enfermeiro envolve o estudo e a prática de diversas competências, de modo a possibilitar a formação de profissionais comprometidos com a saúde e a qualidade de vida da pessoa, família e coletividade, assim como preconizado pelo Código de Ética da profissão. Além disso, o respeito à vida, à dignidade e aos direitos humanos, em todas as dimensões e níveis de complexidade, deve estar sempre presente e ser preservado (COFEN, 2017).

Logo, a formação de uma liga acadêmica mostra-se como uma iniciativa de especial relevância, pois aborda, de maneira efetiva, as referidas prerrogativas, com o fortalecimento do tripé-universitário, a construção do pensamento crítico-reflexivo dos discentes e o envolvimento em atividades para a comunidade (ARAÚJO et al., 2019). A LAEO, mesmo em formato remoto, oferta conteúdos sobre saúde obstétrica e possibilita aos seus ligantes, acadêmicos de enfermagem, o acesso a discussões e reflexões pertinentes acerca do tema proposto, o qual é um diferencial na formação do enfermeiro e de grande relevância para a sociedade. 


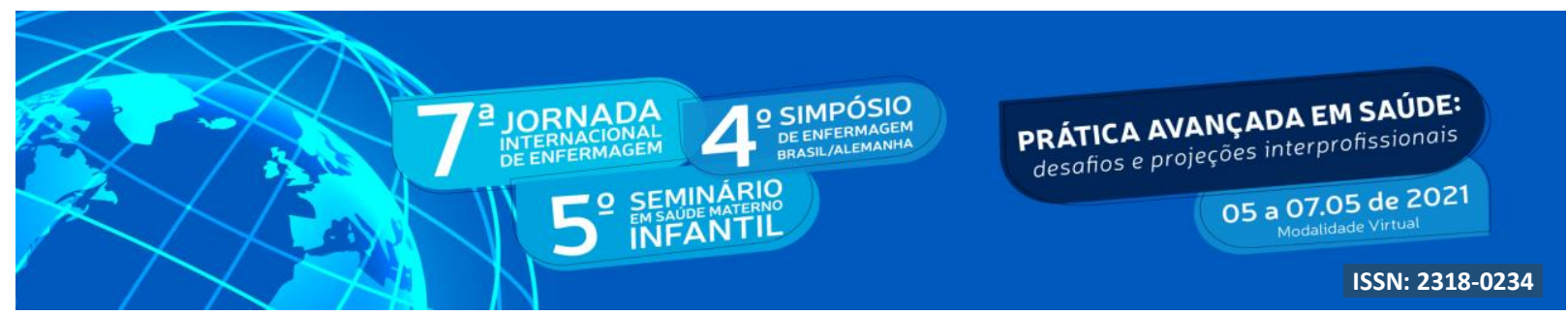

Sob um primeiro momento, a aula inaugural do projeto foi divulgada por meio do Instagram da LAEO. Abordou-se o tema "A residência em enfermagem obstétrica e os caminhos para atuação no exterior", foi transmitida por meio da plataforma de vídeos Youtube e teve, em média, duzentos e cinquenta espectadores. O momento contou com a presença de palestrantes e enfermeiras obstétricas e a primeira discussão foi sobre a relevância e o padrão ouro que é a residência em enfermagem obstétrica, a qual permite ao residente ter práticas constantes na área e obter o título de especialista.

Com isso, foi possível notar, ao analisar os comentários dos participantes no Chat da plataforma, as boas avaliações e grandes expectativas dos estudantes e dos autores para seguir tal tipo de pós-graduação, tendo em vista os exemplos de satisfação tanto profissional quanto pessoal das palestrantes. Outrossim, deu-se bastante ênfase na atuação da enfermagem obstétrica no exterior.

As palestrantes destacaram o fato do Brasil não valorizar de forma adequada o papel do enfermeiro, o que, consequentemente, faz com que alguns busquem por melhores condições de trabalho longe de seu país de origem. Nessa conjuntura, a LAEO permitiu que os participantes refletissem sobre suas perspectivas em relação ao mercado laboral, com relatos e estatísticas sobre as vantagens e desvantagens da profíssão no Brasil e no mundo. Assim, incentivou para que o tempo dos alunos, enquanto acadêmicos de enfermagem, fosse investido em projetos que permitam seu alcance internacional.

O segundo encontro tratou das indicações reais de cesariana. A reunião foi transmitida pelo Youtube, teve em média cento e cinquenta participantes, e a aula ministrada por enfermeiras obstetras e uma doula. Abordou-se, primeiramente, a atual epidemia de cesarianas, na qual o sistema de saúde brasileiro encontra-se, visto que, com a evolução técnica da medicina, o parto passou a ser um fenômeno institucionalizado e, com cada vez menos protagonismo feminino, priorizando outras questões, como o tempo e o lucro.

Além disso, no Brasil, as altas taxas de mortalidade materna e neonatal associam-se com intervenções obstétricas desnecessárias, sendo a cesárea eletiva uma das principais (AYMBERÉ; OLIVEIRA; JÚNIOR, 2020). No entanto, o cuidado da enfermagem obstétrica, no parto, encontra-se associado aos melhores desfechos perinatais, com redução do número de intervenções, como a episiotomia e o parto instrumental, refletindo em uma melhor 


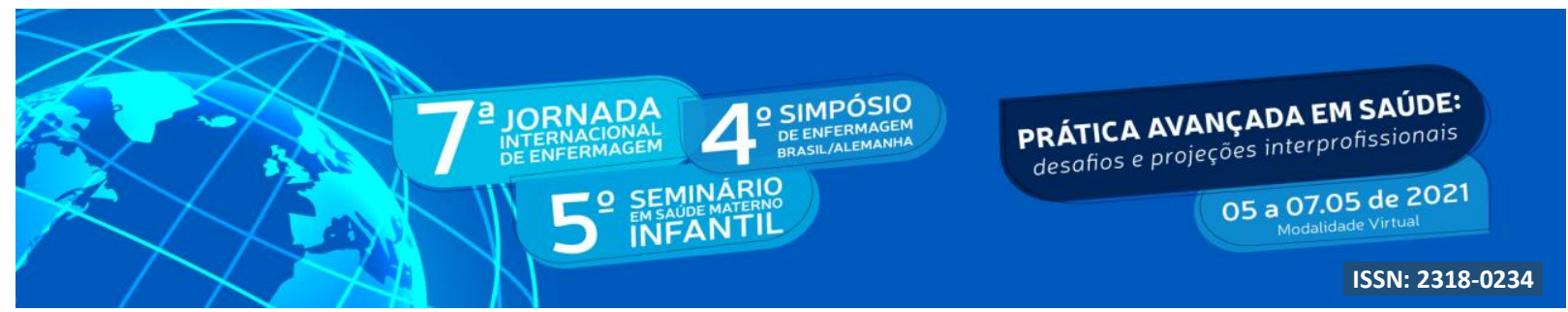

experiência do nascimento para as mães e em maior qualidade em saúde (SILVA et al., 2018).

Assim, de modo análogo, as informações expostas na aula foram de fundamental importância para os autores desse estudo, pois contribuem de forma a esboçar uma reflexão, como futuros profissionais de saúde, para uma visão mais humanizada do gestar e parir, com menos medicalização e tendo a mulher como protagonista desse momento, assim como preconizam as Diretrizes De Atenção à Gestante: A Operação Cesariana (BRASIL, 2016), esclarecendo a parturiente os riscos e repercussões futuras à parturiente de um procedimento deste tipo e encorajando a realização de um parto de via vaginal.

Ademais, esclarecidos os riscos da cesárea para a parturiente e para o bebê, o ligante reflete acerca de seu posterior papel dentro da equipe de saúde, no que tange às reais indicações de cesarianas. Assim, é possível obter, futuramente, uma redução considerável no número desses procedimentos, bem como oferecer às parturientes uma atenção de qualidade e partos com menos riscos, livre de abusos e violências, como preconiza a Política Nacional de Humanização (BRASIL, 2013).

No terceiro encontro, o qual foi de acesso restrito aos ligantes, abordou-se o tema "Aspectos clínicos e epidemiológicos da COVID-19 no ciclo gravídico-puerperal”. Inicialmente, a palestrante, uma enfermeira obstétrica, apresentou um panorama geral da pandemia da COVID-19, discorrendo sobre os sinais, os sintomas e as fases da infecção pelo novo coronavírus, assim como os grupos de risco, do qual as gestantes fazem parte. Posto isso, ponderou-se que grávidas devem seguir todas as orientações de biossegurança, a fim de evitar complicações gestacionais e o desenvolvimento de quadros graves ou até mesmo letais.

Posteriormente, abordou-se os métodos de prevenção, considerando que não há tratamento cientificamente comprovado e eficaz contra a doença. Nessa conjuntura, foi possível verificar que, além de atuar na educação em saúde, estimulando o distanciamento social e o uso de máscaras, a enfermagem constitui-se como protagonista nas ações de proteção e prevenção, ao passo que compõe a maior categoria profissional em saúde (OLIVEIRA et al., 2021).

Quanto ao aleitamento materno, também tratado na aula, a palestrante enfatizou a necessidade que este permaneça mesmo que a mãe esteja infectada com o novo coronavírus. Como futuros profissionais de enfermagem, é de fundamental importância que saibamos 


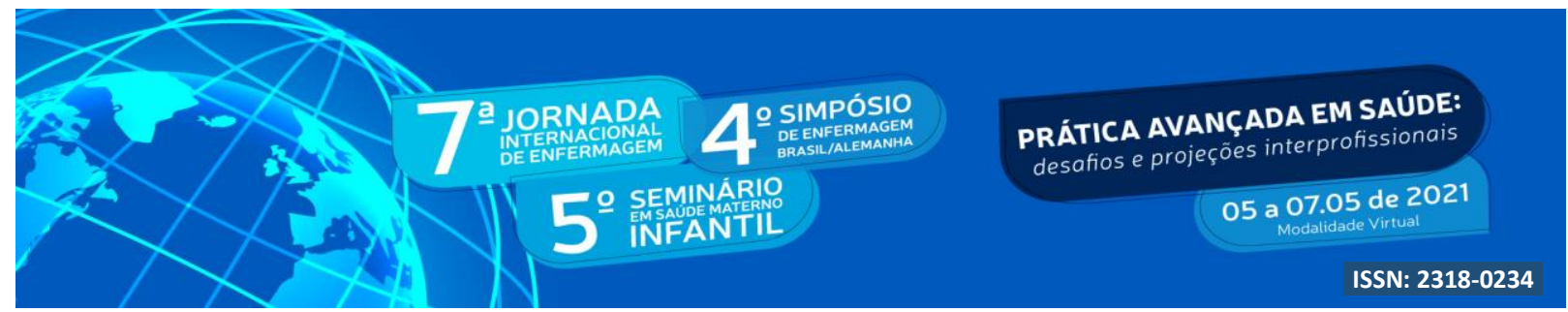

orientar as mães em relação aos benefícios da amamentação, os quais envolvem, de acordo com Braga, Gonçalves e Augusto (2020), a redução de infecções gastrointestinais, melhora da imunidade e a construção de laços afetivos.

Ainda, a Organização Pan-Americana de Saúde (2020), assegura que qualquer risco de infecção do neonato é irrelevante frente essas vantagens, desde que a lactante esteja de máscara a todo momento, lave as mãos com água e sabão antes de tocar na criança e realize a desinfecção do ambiente. Assim, compreende-se a importância do enfermeiro estar sempre atualizado, a fim de prestar o melhor atendimento à mulher, desmistificando possíveis informações falsas.

Já, na quarta aula, discutiu-se a temática "Gestar e parir em tempos de COVID-19". Ministrado por enfermeiras, o evento foi transmitido pelo Youtube e contou com mais de duzentos espectadores. As convidadas relataram suas experiências sobre o período da gravidez e a realização do parto em meio a pandemia da COVID-19, expondo os desafios e medos vivenciados, como: o isolamento; a dificuldade de estabelecer uma rede de apoio de qualidade; a impossibilidade de realizar o plano de parto idealizado; e a vulnerabilidade ao acesso para realização do pré-natal, visto que se expõem a locais de possíveis contaminações.

Tais angústias são justificadas, especialmente, porque além das gestantes se enquadrarem como grupo de risco para a COVID-19, nesse período, também, existem queixas de desrespeito aos direitos da parturiente, como a proibição, por algumas instituições, da presença do acompanhante no momento do parto (ESTRELA et al., 2020). Dessa forma, fazse necessário a criação de estratégias que promovam o bem-estar da mulher nesse momento, considerando as suas fragilidades e demandas, cuidado que é realizado, especialmente, pela equipe de enfermagem (ESTRELA et al., 2020).

Por fim, é importante salientar que também houveram algumas limitações no decorrer das vivências como ligantes da LAEO. As mudanças frequentes dos veículos de transmissão das aulas dificultaram o acesso de uma parte dos ligantes às reuniões, tendo em vista que nem todos estavam habituados às especificidades de cada um deles, o que interfere no processo de aprendizagem. Outrossim, destaca-se que os autores descreveram a experiência de apenas quatro encontros, entretanto, um total de oito ainda estão programados para acontecer, finalizando a participação total na liga. 


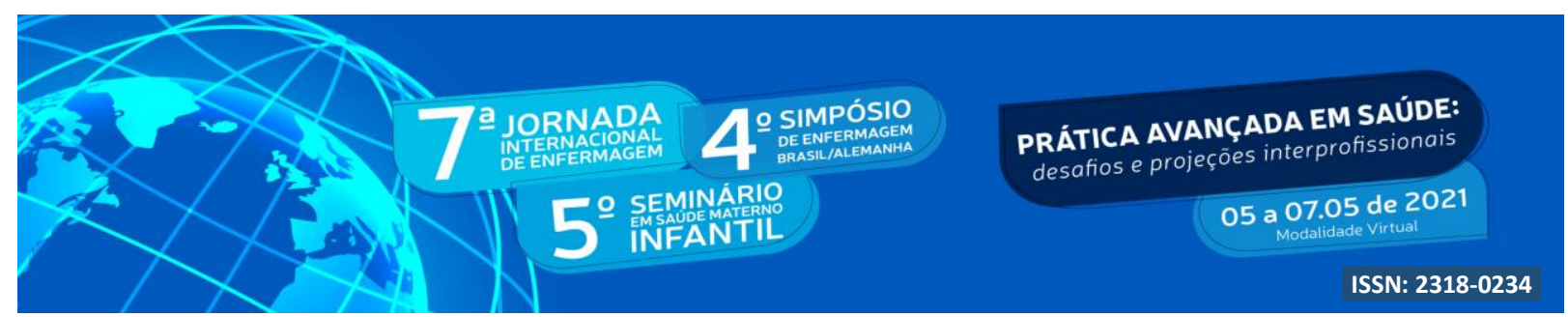

\section{CONCLUSÃO}

De acordo com as vivências relatadas, conclui-se que a LAEO se estabelece como um instrumento de grande impacto na formação e no aprofundamento dos conhecimentos de um futuro profissional da enfermagem, proporcionando o debate de temáticas relevantes para o aluno que almeja inserir-se na obstetrícia desde a graduação. Mesmo com as dificuldades ocasionadas pela modalidade de ensino remoto, todas as aulas e ações realizadas pela Liga, apresentaram uma qualidade ímpar, acrescentando, positivamente, no aprendizado dos discentes de Enfermagem.

Além disso, possibilitou a integração entre cursos e instituições de todo o Brasil, oferecendo um espaço de diálogo, trocas de experiências e construção do conhecimento, possibilitando constantes inovações e atualizações de conceitos e práticas. Nesse sentido, o presente estudo contribui para o entendimento do impacto que as Ligas Acadêmicas possuem para a educação em saúde e, em especial, para a enfermagem.

\section{REFERÊNCIAS}

ARAÚJO, C. R. C., et al. Contribuição das ligas acadêmicas para formação em enfermagem. Revista Enferm. Foco, v. 10, n. 6, p. 137-142, 2019.

AYMBERÉ, A. L.; OLIVEIRA, R. C. A.; JÚNIOR, L. R. G. A importância da enfermagem obstétrica no parto normal. Revista Saúde em Foco, ed. 12, 2020.

BRAGA, M. S.; GONÇALVES, M. S.; AUGUSTO, C. R. Os benefícios do aleitamento materno para o desenvolvimento infantil. Brazilian Journal Of Development, v. 6, n. 9, p. 70250-70261, 2020.

BRASIL, Ministério da Saúde. Diretrizes de Atenção à Gestante: a operação cesariana. Relatório de recomendação, n. 179. Brasília: Conitec, 2016.

BRASIL. Lei n. 9.394 de 20 de dezembro de 1996. Estabelece as diretrizes e bases da educação nacional. Diário Oficial da República Federativa do Brasil. Brasília, 20 dez. 1996. Disponível em: http://www.planalto.gov.br/ccivil_03/leis/19394.htm. Acesso em: 24 mar. 2021.

BRASIL. Ministério da Saúde. Política Nacional De Humanização - HumanizaSus. Brasília, 2013. Disponível em: https://www.gov.br/saude/pt-br/acesso-a-informacao/acoes-eprogramas/humanizasus. Acesso em: 29 de março de 2021. 


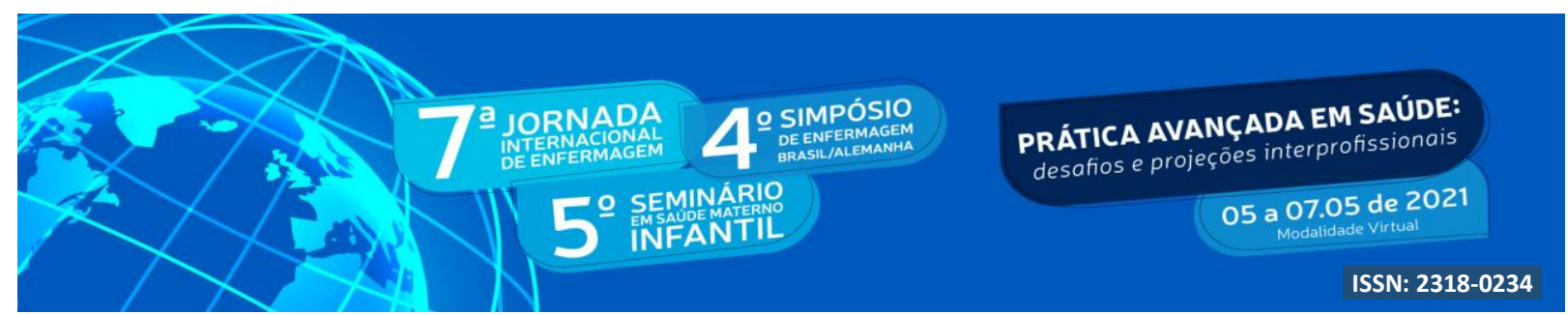

CAETANO, J. R. L. et al. A equipe obstétrica e a parturiente: a problemática da comunicação. Temas em Saúde, v. 16, n.2, p. 283-300, 2016.

CAVALCANTE, A. S. P, et al. As Ligas Acadêmicas na Área da Saúde: Lacunas do Conhecimento na Produção Científica Brasileira. Revista Brasileira de Educação Médica, v. 42, n. 1, p. 197-204, 2018.

COFEN. CONSELHO FEDERAL DE ENFERMAGEM. Resolução n. 564 do Conselho Federal de Enfermagem, 06 de novembro de 2017. Disponível em: http://www.cofen.gov.br/resolucao-cofen-no-5642017_59145.html. Acesso em: 23 mar. 2021.

ESTRELA, F. M., et al. Gestantes no contexto da pandemia da Covid-19: reflexões e desafios. Revista de Saúde Coletiva, v. 30, n.2, p. e300215, 2020.

FERREIRA, E. C.; FALLER, R.J. Relatos do ato de acolher: fragmentos de uma problemática estrutural no acolhimento com classificação de risco. Revista Científica Faculdade Unimed., v. 2, n. 1, p. 81-94, 2020.

LEITE, A. C., et al. Atribuições do enfermeiro no pré-natal de gestantes soropositivas ao HIV atendidas na Unidade Básica De Saúde. Brazilian Journal of Development, v. 6, n. 10, p.78167-78197, 2020.

OLIVEIRA, E. C.; BARBOSA, S. A importância do acompanhamento pré-natal realizado por enfermeiros. Revista Científica FacMais, v. M.; MELO, S. E. P. 7, n. 3, 2016.

OLIVEIRA, K. K. D., et al. Nursing Now e o papel da enfermagem no contexto da pandemia e do trabalho atual. Rev. Gaúcha Enferm., v. 42, p. e20200120, 2021.

OPAS. ORGANIZAÇÃO PAN-AMERICANA DA SAÚDE. Aleitamento materno e a doença causada pelo novo coronavírus (COVID-19). Informações científicas: 23 de junho de 2020. 2020. Disponível em:

https://iris.paho.org/bitstream/handle/10665.2/52479/OPASWBRACOVID-

1920091_por.pdf?sequence=1\&isAllowed=y\&ua=1. Acesso em: 25 mar. 2021.

SILVA, A. F. et al. Atuação do enfermeiro obstetra na assistência ao parto: saberes e práticas humanizadas. Brazilian Journal of Surgery and Clinical Research, v. 23, n. 3, p. 87-93, 2018 .

SILVA, E. A. L.; AMPARO, G. K. S.; SANTOS, E. B. A formação em enfermagem no ciclo gravídico-puerperal. Rev. enferm. UFPE on line, v. 11, n. 12, p. 5139-5144, 2017.

SILVA, S. A.; FLORES, O. Ligas Acadêmicas no Processo de Formação dos Estudantes. Revista Brasileira de Educação Médica, v. 39, n.3, p. 410-425, 2015. 